\title{
Dietary manipulation of histone structure and function
}

\author{
Emily Ho ${ }^{1,2, *}$ and Roderick H. Dashwood ${ }^{2,3}$ \\ ${ }^{1}$ Department of Nutrition \& Exercise Sciences, Oregon State University, Corvallis OR 97331 , \\ USA \\ 2 Linus Pauling Institute, Oregon State University, Corvallis OR 97331, USA \\ 3 Department of Environmental \& Molecular Toxicology, Oregon State University, Corvallis OR \\ 97331, USA
}

\begin{abstract}
The influence of epigenetic alterations during cancer has gained increasing attention over the recent years and has resulted in a paradigm shift in our understanding of mechanisms leading to cancer susceptibility. These features are potentially reversible and may affect genomic stability and expression of genes, including tumor suppressor genes and oncogenes. The reversible acetylation of histones is an important mechanism of gene regulation. Targeting the epigenome, including the use of histone deacetylase (HDAC) inhibitors, is a novel strategy for cancer chemoprevention. We have found that sulforaphane (SFN), a compound found in cruciferous vegetables, inhibits HDAC activity in human colorectal and prostate cancer cells. The ability of SFN to target aberrant acetylation patterns, in addition to effects on phase 2 enzymes, may make it an effective chemoprevention agent. Other dietary agents such as butyrate, allyl sulfides and organoselenium compounds have also shown promise as HDAC inhibitors. These studies are significant because of the potential to qualify or change recommendations for high-risk cancer patients, thereby increasing their survival through simple dietary choices, such as incorporating easily accessible foods into a patient's diet. The work to date provides a scientific foundation for future large-scale human clinical intervention studies with dietary agents that affect the epigenome.
\end{abstract}

\section{Keywords}

histone deacetylase inhibitor; cancer prevention; sulforaphane

\begin{abstract}
Epigenetics is the study of the regulation of gene activity that is not dependent on nucleotide sequence; this may include heritable changes in gene activity and expression, but also longterm alterations in the transcriptional potential of a cell that are not heritable. These features are potentially reversible and may affect genomic stability and expression of genes. In recent years, great strides have been made in understanding the many molecular sequences and patterns that determine which genes can be turned on and off. This work has made it increasingly clear that in addition to genetic changes, the epigenome is just as critical as the DNA sequence itself for healthy human development. Importantly, dietary factors and specific nutrients can modulate epigenetic alterations and alter susceptibility to disease. As the field of epigenetics grows, a whole new level of thinking has emerged as to the impact of nutrients on regulation of gene expression and disease susceptibility. For example, the classic view of cancer etiology is that genetic alterations (via genotoxic agents) damage
\end{abstract}

\footnotetext{
*Correspondence: Emily Ho, PhD, 103 Milam Hall, Dept. of Nutrition \& Exercise Sciences, Oregon State University, Corvallis, OR 97331, USA, Phone: 541-737-9559; fax: 541-737-6914; Emily.ho@oregonstate.edu.
} 
DNA structure and induce mutations resulting in non-functional proteins that lead to disease progression. Aberrant epigenetic events such as DNA hypermethylation and altered histone acetylation have been observed in cancer. To control histone acetylation, a balance exists in normal cells between histone acetyltransferase (HAT) and histone deacetylase (HDAC) activities, and when this balance is disrupted, cancer development can ensue. HDAC activity increases in metastatic cells compared with normal prostate, and global changes in acetylation pattern predicts prostate cancer risk and recurrence [1]. Targeting the epigenome, including the use of HDAC and DNA methyltransferase (DNMT) inhibitors, is an evolving strategy for cancer chemoprevention and both have shown promise in cancer clinical trials [2]. Essential micronutrients such as biotin, B12 and folate, and phytochemicals such as sulforaphane and allyl compounds, can impact epigenetic events as a novel mechanism of action. The goal of this review is to highlight the interactions among nutrients, epigenetics and cancer susceptibility. In particular, we focus on the impact of specific nutrients and food components, such as sulforaphane, on histone modifications that can alter gene expression and influence cancer progression.

\section{Use of histone deacetylase inhibitors in cancer prevention}

Post-translational modifications to histone proteins have been linked to the transcriptional status of chromatin. Modifications of histones include, but are not limited to, phosphorylation, biotinylation, methylation and acetylation. The reversible acetylation of nuclear histones is one of the better characterized histone modifications and is an important mechanism of gene regulation. In general, addition of acetyl groups to histones by HAT enzymes results in an "open" chromatin conformation, facilitating gene expression by allowing transcription factors access to DNA. Removal of acetyl groups by HDACs results in a "closed" conformation, which represses transcription. The HDACs can be divided into four classes based on their structure and sequence homology: class I consists of HDACs 1,2,3, and 8, class II includes HDACs 4,5,6,7,9 and 10, class III enzymes comprise the NAD-dependent Sir2-related proteins, and class IV contains HDAC11. Class I and II HDACs belong to the classical HDACs and their activities are inhibited by trichostatin A (TSA). Class III HDACs are homologous to the yeast Sir2 deacetylases and are a family of proteins classified as sirtuins that are not affected by TSA. Class I HDACs are homologous to the yeast Rpd 3 and are primarily found in nuclear complexes. Class II HDACs are homologous to the yeast protein Hda1, and are capable of translocating in and out of the nucleus. In addition to histone core proteins, several non-histone proteins have been identified that are targeted, especially by Class II HDAC enzymes. Targets include cellular proteins such as transcription factors (e.g., p53, androgen receptor, NFKB), structural (e.g., tubulin) and chaperone proteins (e.g., hsp90), to name a few. Thus, the effects of HDAC inhibitors may be attributed to mechanisms that involve both direct chromatin remodeling and specific modifications to other (non-histone) proteins. When dealing with agents that effect both histone and non-histone acetylation status, the term "KDAC" has been proposed for "lysine deacetylase" inhibitors (the letter " $\mathrm{K}$ " being the biochemical abbreviation for lysine).

Increased HDAC activity and expression is common in many cancer malignancies, and can result in repression of transcription that results in a de-regulation of differentiation status, cell cycle checkpoint controls, and apoptotic mechanisms. Moreover, tumor suppressor genes, such as $p 21$ appear to be targets of HDACs and are "turned off" by deacetylation. Prostate cancer cells also exhibit aberrant acetylation patterns. In human patient samples, global decreases in histone acetylation state corresponded with increased grade of cancer and risk of prostate cancer recurrence [1]. Importantly, inhibitors of HDAC, including suberoylanilide hydroxamic acid (SAHA), valproic acid, depsipeptide, and sodium butyrate have been demonstrated to be effective against prostate cancer cell lines and xenograft 
models $[3,4]$. Specific genes associated with prostate cancer such as tubulin, coxsackie and adenovirus receptor (CAR), liver cancer-1 (DLC-1) and KLF-6 have also shown to be hypoacetylated and repressed in prostate cancers [5-7]. The use of Class I and Class II HDAC inhibitors in cancer chemoprevention and therapy has gained significant interest. Several clinical trials are currently ongoing aimed at establishing the chemotherapeutic efficacy of HDAC inhibitors, based on evidence that cancer cells undergo cell cycle arrest, differentiation and apoptosis in vitro, and that tumor volume and/or tumor number may be reduced in animal models. HDAC inhibitors have been shown to increase global acetylation as well as acetylation associated with specific gene promoters. Although the equilibrium is shifted toward greater histone acetylation after treatment with HDAC inhibitors, the expression of only a relatively small number of genes is altered in an upward or downward direction [8]. Importantly, only neoplastically transformed cells appear to respond to increased acetylation by undergoing differentiation, cell cycle arrest, or apoptosis; normal cells, despite the increased acetylation, do not respond in this manner to HDAC inhibitors [9]. Thus, effects of HDAC inhibitors on apoptosis and anti-proliferation appear to be selective to cancer, not normal cells, although the mechanism is poorly understood. In general, HDAC inhibitors have been sub-divided into several classifications; short chain fatty acids, hydroxamic acids, cyclic tetrapeptides, and benzamides [10,11]. Most have a conserved structure and act by blocking the HDAC catalytic site. Many of these pharmacological HDAC inhibitors have been used in phase I and phase I/II clinical trials with promising results [12]. However, many of these compounds also exhibit several associated side-effects and toxicities. For example, valproic acid and trichostatin A have been associated with developmental abnormalities such as neural tube defects [13]. The use of SAHA has also been associated with several hematologic toxicities such as myelosuppression and thrombocytopenia [14]. Many of these drugs must also be administered i.v., a less than ideal route of administration for patients. Although there has been some attempt to develop oral HDAC inhibitor drugs, these also have side-effects such as fatigue, anorexia, dehydration and GI upset $[14,15]$. The identification of HDAC inhibitors, with low toxicity but therapeutic efficacy, is an important area of research.

\section{Dietary inhibitors of histone deacetylases}

Recent studies also suggest that sulforaphane (SFN), an isothiocyanate derived from cruciferous vegetables, is an inhibitor of HDAC activity and offers protection against tumor development during the "post-initiation" phase of carcinogenesis. The general structure of HDAC inhibitors is comprised of a functional group at one end that interacts with a zinc atom and neighboring amino acids at the base of the HDAC active site, a spacer that fits into the channel of the active site, and a cap group which is hypothesized to interact with external amino acid residues $[16,17]$. Based on the similarity of SFN metabolites to the conserved structure of HDAC inhibitors, we hypothesized that SFN could effectively inhibit HDAC activity. SFN is metabolized via the mercapturic acid pathway, starting with glutathione (GSH) conjugation by glutathione- $S$-transferase (GST) and subsequent steps generate SFN-cysteine (SFN-Cys) followed by SFN- $N$-acetylcysteine (SFN-NAC). Based on modeling and in vitro work [18-20], it has been hypothesized that SFN-NAC or SFNCys are the active HDAC inhibitors. This was supported by metabolite studies, showing significant levels of SFN-Cys generated in SFN-treated prostate cancer cells (J. Clark and E. Ho, unpublished data). Molecular modeling in the active site of an HDAC enzyme provided evidence that SFN-Cys docked in the HDAC pocket as a competitive inhibitor [21]. In BPH1, PC3, and LnCap prostate cancer cells, SFN inhibited HDAC activity with a concomitant increase in global histone acetylation, increased acetylated histone $\mathrm{H} 4$ interactions with the $P 21$ and Bax promoter, and induction of p21 and Bax mRNA and protein levels [22]. SFN also decreased the expression of HDAC6, a Class II HDAC and induced concomitant increases in acetyl-tubulin levels (unpublished data). HDAC inhibition 
coincided with the induction of $\mathrm{G}_{2} / \mathrm{M}$ phase cell cycle arrest and apoptosis, as indicated by multi-caspase activation [22]. HDAC inhibition by SFN has also been established in several other cancer cell lines including breast and colon [21,23], suggesting the effects are not specific to the prostate. In HCT116 human colorectal cancer cells treated with SFN there were decreases in HDAC activity, increased global histone acetylation, and a selective increase in histone acetylation at the $p 21$ promoter [21]. HT29 colon cancer cells, which lack endogenous Nrf2 protein, as well as $\mathrm{Nrf}^{-1-}$ mouse embryonic fibroblasts, both exhibited an HDAC inhibitory response to SFN treatment. These results indicated the possibility of a separate SFN chemoprevention pathway distinct from the classic Nrf2 pathway [24]. Importantly, the effects of SFN do appear to be tumor cell specific. We have found that 3-15 $\mu \mathrm{M}$ SFN induces potent HDAC inhibition and G2/M arrest in PC3 cancer cells, but have no effect on normal prostate epithelial cells (unpublished data). These data support the hypothesis that HDAC inhibition may be an important mechanism of chemoprevention for SFN and similar pharmacological HDAC inhibitors, the cytotoxic effects are specific to cancer, not normal cells.

In vivo, dietary SFN supplementation resulted in slower tumor growth and significant HDAC inhibition in the PC3 xenografts, as well as HDAC inhibition in the prostate and circulating peripheral blood mononuclear cells [25]. In other dietary studies examining intestinal cancer, $A p c^{\mathrm{min}}$ mice were fed $\sim 6 \mu \mathrm{mol}$ SFN/day for 10 weeks. In these experiments a significant decrease in intestinal polyps and an increase in global acetylated histones $\mathrm{H} 3$ and $\mathrm{H} 4$ were observed, with specific increases at the Bax and $p 21$ promoters [26]. From these studies it can be concluded that HDAC inhibition represents a novel chemoprevention mechanism by which SFN might promote cell cycle arrest and apoptosis in vivo. To date very few human clinical trials have evaluated the effects of SFN on cancer outcome; however, several pilot and phase I human SFN trials have been conducted utilizing different sources of SFN. In our laboratory, a small intervention study was performed to determine if the HDAC inhibition effects observed in cell culture and mice could be translated into humans. In clinical trials using pharmacological HDAC inhibitors such as SAHA, alterations in acetylated histone status in peripheral blood cell samples are used as a biomarker for HDAC inhibitory efficacy. In normal healthy volunteers, after the ingestion of $68 \mathrm{~g}$ of broccoli sprouts, a significant decrease in HDAC activity was evident in peripheral blood mononuclear cells with a concomitant increase in acetylated histones $\mathrm{H} 3$ and $\mathrm{H} 4$ [25]. Broccoli sprouts are a rich source of glucoraphanin, the precursor of SFN; thus, these data give preliminary evidence for the ability of dietary SFN to inhibit HDAC activity in humans. Follow-up studies will examine the relationship between specific SFN metabolites in the circulation and HDAC inhibition.

In addition to SFN, there are many other known and putative diet-derived HDAC inhibitors. Experiments with structurally-related isothiocyanates such as sulforaphene, erucin, and phenylbutyl isothiocyanate, had comparable HDAC inhibitory activities [20]. Butyrate is the smallest known HDAC inhibitor (reviewed in [27]), and contains a simple three carbon 'spacer' attached to a carboxylic acid group. This compound is derived from the fermentation of dietary fiber and represents the primary metabolic fuel for the colonocytes, where it is present at millimolar concentrations. Recent studies have confirmed that butyrate acts as a competitive HDAC inhibitor [28]. A second class of dietary agent reported to inhibit HDAC activity in vitro is the garlic organosulfur compounds, such as DADS and $S$ allylmercaptocysteine [29], which can be metabolized to allyl mercaptan (AM), a competitive HDAC inhibitor [29]. Treatment of human colon cancer cells with AM induced rapid histone acetylation along with HDAC inhibition, resulting in increased association of acetylated histones and $\mathrm{Sp} 3$ transcription factor binding to the promoter element of P21Wafl, thereby increasing both p21 mRNA and protein expression and triggering cell cycle arrest [30]. More recently, $\alpha$-keto acid metabolites of organoselenium compounds 
have also been identified as novel HDAC inhibitors in both colon and prostate cancer cells. In particular, the metabolite methylselenopyruvate (MSP) caused HDAC inhibition, increases in acetylated histone and p21 promoter activity, and concomitant increases in apoptosis and cell cycle arrest at concentrations as low as $2 \mu \mathrm{M}([31,32])$.

\section{Future directions and conclusions}

In addition to histone modifications, methylation of $\mathrm{CpG}$ islands in promoter elements is a major epigenetic controlling event for gene silencing [33-35]. In fact, transcriptional silencing by aberrant hypermethylation of $\mathrm{CpG}$ islands has been reported in nearly every tumor type [36, 37]. Many of the commonly silenced genes include tumor suppressor genes and genes involved in carcinogen detoxification, hormonal responses and cell cycle control [37-40]. Both DNA hypermethylation and histone modifications are closely related aspects of chromatin remodeling. Epigenetic control of gene expression often requires the cooperation and interaction of both mechanisms, and disruption in these processes can lead to genomic instability and gene silencing, resulting in cancer progression. Interestingly, DNMT1 also appears to direct histone modifications by recruiting HDACs [41]. Methylation of CpG sequences by DNMT1 binds specific methylated DNA binding (MBD) proteins such as MeCP2 and MBD2. This MBD binding complex recruits a complex of transcriptional repressors, including HDACs, which results in chromatin-associated gene silencing $[42,43]$. This relationship between DNA methylation and chromatin remodeling suggests significant cross-talk among distinct epigenetic pathways that control gene silencing/unsilencing. Indeed, the combination of pharmacological DNMT inhibitors and HDAC inhibitors has been explored as a potential anti-tumor therapy [44, 45]. However, DNMT inhibitor drugs have potential hazards and side effects because they often require incorporation into DNA, thereby targeting cells dividing in S phase, leading to greater toxicity [46, 47]. Recently, dietary agents that have dual action of promoter methylation and HDAC inhibition have been identified. Phenethyl isothiocyanate (PEITC), an isothiocyanate related to SFN and which is found in cruciferous vegetables such as watercress, was shown to reverse hypermethylation of GSTP1 promoter elements in androgen-dependent and androgen-independent prostate cancer cells. Concurrent with demethylation effects, PEITC $(2-5 \mu \mathrm{M})$ inhibited HDAC activity and increase acetylated histone status. At the doses tested, PEITC was more effective towards promoter demethylation and HDAC inhibition than chemical DNMT and HDAC inhibitors, 5-aza and TSA [48]. Different epigenetic modifications clearly appear to work together to coordinate and maintain gene expression patterns in the cell. Further work examining the possible cross-talk between various epigenetic modifications after exposure to dietary epigenetic modulators appears to be warranted.

Overall, the identification of dietary agents that target HDAC and/or DNA methylation, with few side effects, is an important area of research (reviewed in [20, 49, 50]), and aligns with the NIH Roadmap priority area on 'epigenetics'. Many of these dietary agents have multiple actions on various pathways during carcinogenesis, and their ability to target several mechanisms, including epigenetic targets, may increase their efficacy as chemoprevention agents. Further, the use of dietary strategies to inhibit HDACs or other epigenetic modifiers as chemoprevention agents is significant because of the ease of implementation into clinical trials, due to their relatively non-toxic nature. Ultimately, these types of study have the potential to decrease prevalence of various cancers and/or increase survival through simple dietary choices, such as incorporating easily accessible foods into a patient's diet.

\section{Acknowledgments}

This work was supported in part by the Oregon Agricultural Experiment Station, supported in part by funds provided through the Hatch Act. Additional support was provided by NIH grants CA090890, CA065525, 
CA122906, CA122959 and Environmental Health Sciences Center (National Institute of Environmental Health Sciences) P30 ES00210.

\section{References}

1. Seligson DB, Horvath S, Shi T, et al. Global histone modification patterns predict risk of prostate cancer recurrence. Nature. 2005; 435:1262-6. [PubMed: 15988529]

2. Sigalotti L, Fratta E, Coral S, et al. Epigenetic drugs as pleiotropic agents in cancer treatment: biomolecular aspects and clinical applications. J Cell Physiol. 2007; 212:330-44. [PubMed: 17458893]

3. Fronsdal K, Saatcioglu F. Histone deacetylase inhibitors differentially mediate apoptosis in prostate cancer cells. Prostate. 2004

4. Butler LM, Agus DB, Scher HI, et al. Suberoylanilide Hydroxamic Acid, an Inhibitor of Histone Deacetylase, Suppresses the Growth of Prostate Cancer Cells in Vitro and in Vivo. Cancer Res. 2000; 60:5165-5170. [PubMed: 11016644]

5. Li D, Yea S, Dolios G, et al. Regulation of Kruppel-like factor 6 tumor suppressor activity by acetylation. Cancer Res. 2005; 65:9216-25. [PubMed: 16230382]

6. Guan M, Zhou X, Soulitzis N, Spandidos DA, Popescu NC. Aberrant methylation and deacetylation of deleted in liver cancer-1 gene in prostate cancer: potential clinical applications. Clin Cancer Res. 2006; 12:1412-9. [PubMed: 16533763]

7. Soucek K, Kamaid A, Phung AD, et al. Normal and prostate cancer cells display distinct molecular profiles of alpha-tubulin posttranslational modifications. Prostate. 2006; 66:954-65. [PubMed: 16541425]

8. Mitsiades CS, Mitsiades NS, McMullan CJ, et al. Transcriptional signature of histone deacetylase inhibition in multiple myeloma: biological and clinical implications. Proc Natl Acad Sci U S A. 2004; 101:540-5. [PubMed: 14695887]

9. Brinkmann H, Dahler AL, Popa C, et al. Histone hyperacetylation induced by histone deacetylase inhibitors is not sufficient to cause growth inhibition in human dermal fibroblasts. J Biol Chem. 2001; 276:22491-9. [PubMed: 11304533]

10. Vannini A, Volpari C, Filocamo G, et al. Crystal structure of a eukaryotic zinc-dependent histone deacetylase, human HDAC8, complexed with a hydroxamic acid inhibitor. Proc Natl Acad Sci U S A. 2004; 101:15064-9. [PubMed: 15477595]

11. Balasubramanian S, Verner E, Buggy JJ. Isoform-specific histone deacetylase inhibitors: the next step? Cancer Lett. 2009; 280:211-21. [PubMed: 19289255]

12. Garcia-Manero G, Issa JP. Histone deacetylase inhibitors: a review of their clinical status as antineoplastic agents. Cancer Invest. 2005; 23:635-42. [PubMed: 16305991]

13. Wiltse J. Mode of action: inhibition of histone deacetylase, altering WNT-dependent gene expression, and regulation of beta-catenin--developmental effects of valproic acid. Crit Rev Toxicol. 2005; 35:727-38. [PubMed: 16417040]

14. O'Connor OA, Heaney ML, Schwartz L, et al. Clinical experience with intravenous and oral formulations of the novel histone deacetylase inhibitor suberoylanilide hydroxamic acid in patients with advanced hematologic malignancies. J Clin Oncol. 2006; 24:166-73. [PubMed: 16330674]

15. Kelly WK, O'Connor OA, Krug LM, et al. Phase I study of an oral histone deacetylase inhibitor, suberoylanilide hydroxamic acid, in patients with advanced cancer. J Clin Oncol. 2005; 23:392331. [PubMed: 15897550]

16. Finnin MS, Donigian JR, Cohen A, et al. Structures of a histone deacetylase homologue bound to the TSA and SAHA inhibitors. Nature. 1999; 401:188-93. [PubMed: 10490031]

17. Furumai R, Komatsu Y, Nishino N, Khochbin S, Yoshida M, Horinouchi S. Potent histone deacetylase inhibitors built from trichostatin A and cyclic tetrapeptide antibiotics including trapoxin. Proc Natl Acad Sci U S A. 2001; 98:87-92. [PubMed: 11134513]

18. Dashwood RH, Ho E. Dietary histone deacetylase inhibitors: From cells to mice to man. Semin Cancer Biol. 2007 
19. Myzak MC, Karplus PA, Chung F-L, Dashwood RH. A Novel Mechanism of Chemoprotection by Sulforaphane: Inhibition of Histone Deacetylase. Cancer Res. 2004; 64:5767-5774. [PubMed: 15313918]

20. Myzak MC, Ho E, Dashwood RH. Dietary agents as histone deacetylase inhibitors. Mol Carcinog. 2006; 45:443-6. [PubMed: 16652377]

21. Myzak MC, Karplus PA, Chung FL, Dashwood RH. A novel mechanism of chemoprotection by sulforaphane: inhibition of histone deacetylase. Cancer Res. 2004; 64:5767-74. [PubMed: 15313918]

22. Myzak MC, Hardin K, Wang R, Dashwood RH, Ho E. Sulforaphane inhibits histone deacetylase activity in BPH-1, LnCaP and PC-3 prostate epithelial cells. Carcinogenesis. 2006; 27:811-819. [PubMed: 16280330]

23. Pledgie-Tracy A, Sobolewski MD, Davidson NE. Sulforaphane induces cell type-specific apoptosis in human breast cancer cell lines. Mol Cancer Ther. 2007; 6:1013-21. [PubMed: 17339367]

24. Dashwood RH, Ho E. Dietary histone deacetylase inhibitors: From cells to mice to man. Semin Cancer Biol. 2007; 17:363-9. [PubMed: 17555985]

25. Myzak MC, Tong P, Dashwood WM, Dashwood RH, Ho E. Sulforaphane retards the growth of human PC-3 xenografts and inhibits HDAC activity in human subjects. Exp Biol Med (Maywood). 2007; 232:227-34. [PubMed: 17259330]

26. Myzak MC, Dashwood WM, Orner GA, Ho E, Dashwood RH. Sulforaphane inhibits histone deacetylase in vivo and suppresses tumorigenesis in Apc-minus mice. Faseb J. 2006; 20:506-8. [PubMed: 16407454]

27. Davie JR. Inhibition of histone deacetylase activity by butyrate. J Nutr. 2003; 133:2485S-2493S. [PubMed: 12840228]

28. Sekhavat A, Sun JM, Davie JR. Competitive inhibition of histone deacetylase activity by trichostatin A and butyrate. Biochem Cell Biol. 2007; 85:751-8. [PubMed: 18059533]

29. Druesne N, Pagniez A, Mayeur C, et al. Diallyl disulfide (DADS) increases histone acetylation and p21(waf1/cip1) expression in human colon tumor cell lines. Carcinogenesis. 2004; 25:1227-36. [PubMed: 14976134]

30. Nian H, Delage B, Pinto JT, Dashwood RH. Allyl mercaptan, a garlic-derived organosulfur compound, inhibits histone deacetylase and enhances Sp3 binding on the P21WAF1 promoter. Carcinogenesis. 2008; 29:1816-24. [PubMed: 18628250]

31. Lee JI, Nian H, Cooper AJ, et al. Alpha-keto acid metabolites of naturally occurring organoselenium compounds as inhibitors of histone deacetylase in human prostate cancer cells. Cancer Prev Res (Phila Pa). 2009; 2:683-93.

32. Nian H, Bisson WH, Dashwood WM, Pinto JT, Dashwood RH. Alpha-keto acid metabolites of organoselenium compounds inhibit histone deacetylase activity in human colon cancer cells. Carcinogenesis. 2009; 30:1416-23. [PubMed: 19528666]

33. Turker MS, Bestor TH. Formation of methylation patterns in the mammalian genome. Mutat Res. 1997; 386:119-30. [PubMed: 9113113]

34. Jones PA, Baylin SB. The fundamental role of epigenetic events in cancer. Nat Rev Genet. 2002; 3:415-28. [PubMed: 12042769]

35. Wolffe AP, Matzke MA. Epigenetics: regulation through repression. Science. 1999; 286:481-6. [PubMed: 10521337]

36. Baylin SB. DNA methylation and gene silencing in cancer. Nat Clin Pract Oncol. 2005; 2 (Suppl 1):S4-11. [PubMed: 16341240]

37. Baylin SB, Esteller M, Rountree MR, Bachman KE, Schuebel K, Herman JG. Aberrant patterns of DNA methylation, chromatin formation and gene expression in cancer. Hum Mol Genet. 2001; 10:687-92. [PubMed: 11257100]

38. Herman JG, Baylin SB. Gene silencing in cancer in association with promoter hypermethylation. $\mathrm{N}$ Engl J Med. 2003; 349:2042-54. [PubMed: 14627790]

39. Baylin SB, Ohm JE. Epigenetic gene silencing in cancer - a mechanism for early oncogenic pathway addiction? Nat Rev Cancer. 2006; 6:107-16. [PubMed: 16491070] 
40. Li LC, Carroll PR, Dahiya R. Epigenetic changes in prostate cancer: implication for diagnosis and treatment. J Natl Cancer Inst. 2005; 97:103-15. [PubMed: 15657340]

41. Dobosy JR, Selker EU. Emerging connections between DNA methylation and histone acetylation. Cell Mol Life Sci. 2001; 58:721-7. [PubMed: 11437233]

42. $\mathrm{Ng} \mathrm{HH}$, Zhang Y, Hendrich B, et al. MBD2 is a transcriptional repressor belonging to the MeCP1 histone deacetylase complex. Nat Genet. 1999; 23:58-61. [PubMed: 10471499]

43. Fuks F, Burgers WA, Brehm A, Hughes-Davies L, Kouzarides T. DNA methyltransferase Dnmt1 associates with histone deacetylase activity. Nat Genet. 2000; 24:88-91. [PubMed: 10615135]

44. Gore SD, Baylin S, Sugar E, et al. Combined DNA methyltransferase and histone deacetylase inhibition in the treatment of myeloid neoplasms. Cancer Res. 2006; 66:6361-9. [PubMed: 16778214]

45. Cameron EE, Bachman KE, Myohanen S, Herman JG, Baylin SB. Synergy of demethylation and histone deacetylase inhibition in the re-expression of genes silenced in cancer. Nat Genet. 1999; 21:103-7. [PubMed: 9916800]

46. Ghoshal K, Bai S. DNA methyltransferases as targets for cancer therapy. Drugs Today (Barc). 2007; 43:395-422. [PubMed: 17612710]

47. Juttermann R, Li E, Jaenisch R. Toxicity of 5-aza-2'-deoxycytidine to mammalian cells is mediated primarily by covalent trapping of DNA methyltransferase rather than DNA demethylation. Proc Natl Acad Sci U S A. 1994; 91:11797-801. [PubMed: 7527544]

48. Wang LG, Beklemisheva A, Liu XM, Ferrari AC, Feng J, Chiao JW. Dual action on promoter demethylation and chromatin by an isothiocyanate restored GSTP1 silenced in prostate cancer. Mol Carcinog. 2007; 46:24-31. [PubMed: 16921492]

49. Myzak MC, Dashwood RH. Chemoprotection by sulforaphane: keep one eye beyond Keap1. Cancer Lett. 2006; 233:208-18. [PubMed: 16520150]

50. Nian H, Delage B, Ho E, Dashwood RH. Modulation of histone deacetylase activity by dietary isothiocyanates and allyl sulfides: studies with sulforaphane and garlic organosulfur compounds. Environ Mol Mutagen. 2009; 50:213-21. [PubMed: 19197985] 